Med Klin Intensivmed Notfmed 2013.

108:548-548

DOI 10.1007/s00063-012-0138-6

Online publiziert: 4. September 2013

๑) Springer-Verlag Berlin Heidelberg 2013

G. Heinz ${ }^{1}$ A. Geppert ${ }^{2}$

${ }^{1}$ Abteilung für Kardiologie, Intensivstation 13H3, Universitätsklinik für Innere Medizin II, Wien

${ }^{2}$ Kardiologie, Herzintensivstation, Wilhelminenspital der Stadt Wien

\title{
Spezielle kardiologische Probleme auf der Intensivstation
}

Liebe Kolleginnen und Kollegen, liebe Leserinnen und Leser,

als Intensivmediziner mit Schwerpunkt in der Behandlung kardiovaskulärer Erkrankungen sehen wir uns an der Schnittstelle von Intensivmedizin, Kardiologie, Kardioanästhesie und Kardiochirurgie. In der Kardiologie wie in der Intensivmedizin wurden in den letzten Jahren große Fortschritte gemacht. Nicht zuletzt aufgrund einer zunehmend älteren Bevölkerung mit steigender Inzidenz an degenerativen Herzerkrankungen finden sich immer mehr Patienten wegen oder mit einer Herzerkrankung auf der Intensivstation ein. Diesem speziellen Thema möchte sich dieser Sonderband widmen.

Scheinbar einfach - mit Vorhofflimmern - fängt es an. Heute ist bekannt, dass Vorhofflimmern die häufigste, ja schlechthin die Rhythmusstörung auf der Intensivstation ist. Was können wir daraus ableiten, und welche Konsequenzen hat dies? Wir haben heute Hinweise, dass sich Vorhofflimmern auf jeden Fall bei postoperativen, vielleicht auch bei kritisch Kranken im Allgemeinen, etwas anders verhält als in der durchschnittlichen Bevölkerung. Es ist schwerer zu behandeln, und scheint - so therapieresistent es während der Phase des kritisch Krankseins auch ist - oft auf diese kritische Phase beschränkt zu sein. Danach ist es offenbar kein Problem mehr.

Oft ist es nicht einfach zu unterscheiden, ob der Patient mit oder durch eine kardiologische Grundkrankheit, etwa eine Herzinsuffizienz oder eine Herzklappenerkrankung, akut kritisch krank geworden ist. Diesem sehr schwierigen
Thema, das viel Erfahrung erfordert, widmet sich Herr Geppert in einem ausführlichen Beitrag.

Schließlich sehen wir heute auch zunehmend häufiger Patienten mit angeborenen Herzfehlern in der Anästhesiologie und auf der Intensivstation (GrownUp Congenital Heart Disease, GUCH). Unsere Gastautorin Frau Dominique Bettex versteht es in einzigartiger Weise, in ihrem Beitrag dieses sehr komplexe Thema verständlich darzustellen, ohne bei Ihnen, liebe Leserin, lieber Leser, umfangreiche Vorkenntnisse der vielen komplexen Herzfehler vorauszusetzen. Man spürt ihre jahrzehntelange Expertise im Umgang mit diesem Patientenkollektiv.

Und Hand auf's Herz: haben Sie noch nie vor Rhythmusstörungen, noch schlimmer: vor Patienten mit Rhythmusstörungen und implantiertem Defibrillator (ICD), auf Ihrer Intensivstation Sorge gehabt? Kollege Gatterer, ambitionierter Rhythmologe und Intensivmediziner, erklärt Ihnen in seinem Beitrag alles, was an kontemporärer Gerätetechnologie derzeit verfügbar ist und wie Sie damit umgehen.

Natürlich konnten wir nicht alle Aspekte kardiologischer Patienten auf der Intensivstation abhandeln. Wir wünschen uns dennoch, dass Sie unseren Sonderband oft zur Hand nehmen und dass er Ihnen in Ihrer Praxis eine wertvolle Hilfe und Anleitung sein möge.
Herzlichst, auch im Namen aller Autoren Ihr

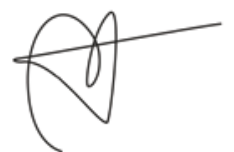

Ao. Univ. Prof. Dr. Gottfried Heinz

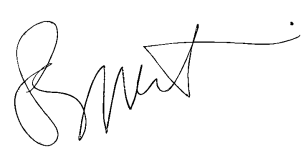

Univ. Doz. Dr. Alexander Geppert

\section{Korrespondenzadressen}

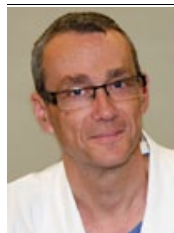

Ao. Univ. Prof. Dr. G. Heinz

Abteilung für Kardiologie, Intensivstation $13 \mathrm{H} 3$, Universitätsklinik für Innere Medizin II Währinger Gürtel 18-20, 1090 Wien Österreich gottfried.heinz@ meduniwien.ac.at

Univ. Doz. Dr. A. Geppert
Kardiologie,
Herzintensivstation,
Wilhelminenspital der
Stadt Wien
1160 Wien
Österreich
alexander.geppert@wienkav.at

\section{Einhaltung der ethischen Richtlinien}

Interessenkonflikt. G. Heinz und A. Geppert geben an, dass kein Interessenkonflikt besteht. 